\title{
Motion-compensated prediction based algorithm for medical image sequence compression ${ }^{\wedge}$
}

\author{
Seyfullah H. Oǧuz, Ömer N. Gerek*, A. Enis Çetin \\ Department of Electrical and Electronics Engineering, Bilkent University, Bilkent, Ankara 06533, Turkey
}

Received 15 November 1993

\begin{abstract}
A method for irreversible compression of medical image sequences is described. The method relies on discrete cosine transform and motion-compensated prediction to reduce intra- and inter-frame redundancies in medical image sequences. Simulation examples are presented.
\end{abstract}

\section{Introduction}

Digital medical images are produced by a number of medical imaging devices including computed tomography (CT), magnetic resonance imaging (MRI), ultrasound, digital radiography, and radiographic film digitizers. Due to the large amount of data per image there is a storage problem in medical Picture Archiving and Communication Systems (PACS) $[5,12]$. Image compression is an efficient way of handling this problem $[5,12,2,4,7,9,13]$. Reversible (lossless) image compression techniques usually achieve a degree of compression in the order of $3: 1$, whereas irreversible (lossy) techniques may achieve compression ratios in the order of

\footnotetext{
This work is supported by TUBITAK - Scientific and Technical Research Council of Turkey, and it was presented in the "Canadian Conference on Electrical and Computer Engineering", September 1992.

* Corresponding author.
}

$5: 1$ to $20: 1$. Irreversible techniques which do not introduce any visual degradation can be used in a PACS [5].

Most of the irreversible image coding techniques use transform domain methods. For example, discrete cosine transform (DCT) based techniques have been successfully employed for still picture coding including medical image compression, and video coding in practice $[12,4,2,7,9,13,10,14]$.

Many medical imaging devices, including the CT and MRI machines, produce image sequences. In a typical radiology department $20-30 \%$ of all data created are image sequences. Regular medical image sequences contain images of slices of the human body. Therefore, neighboring images in a CT or an MRI sequence are highly correlated with each other. In order to compress such a medical image sequence, one should not only consider intra-frame coding but also exploit the high correlation in the third dimension to achieve further compression. 
This paper presents a three-dimensional (3-D) image compression algorithm which takes advantage of the correlation among the neighboring images. The algorithm relies on DCT-based compression within a medical image and 'motion-compensated' prediction [6] to remove redundancies between images in the sequence.

\section{Image sequence coding algorithm}

One of the key features of our algorithm is the use of 'motion compensation' which is utilized to remove the redundancies between image frames. Although there may not be real motion in most medical image sequences, one can also consider the changes occurring in consecutive images of the sequence as motion of the blocks and treat accordingly.

In essence, our algorithm is similar to the MPEG-I standard [8]. However, the MPEG-I standard is not suitable for coding medical image sequences directly. This standard is commonly used for digital video with 8 bits per pixel luminance and chrominance components. On the other hand, our algorithm is aimed to compress gray level medical image sequences with 10 and 12 bits per pixel resolutions. Moreover, the features of medical images are different from the regular video sequences. Based on the statistical analysis of the quantized transform coefficients, we designed a new variablelength codeword (VLC) tables for coding (run, level) pairs.

The syntax of our algorithm consists of a fivelayered hierarchical structure for the coded bit stream. These layers are also present in the MPEG-I standard, but with a different syntax and for different information. Each layer supports a set of functions which are essential for our coding purposes.

The first layer in this hierarchical structure is the sequence layer. Just like in the standard MPEG-I, the sequence header contains information to distinguish the image set of one patient from another one. This header contains data regarding the patient characteristics together with the necessary coding informations such as horizontal and vertical picture resolutions, pixel aspect ratios, and quantization.

The second layer is the group of pictures (GOP) layer which is essential for our algorithm. The ran- dom access points are determined by the GOP layer header. These headers may also contain information such as the characteristics of the included slice images. The corresponding body places of the slice images contained in the GOP can also be written in the GOP header.

The third layer is the picture layer. In the proposed method, the coded medical image sequence consists of I-type pictures (intra-frame coded) and P-type pictures (inter-frame coded) as described in [1].

The slice layer concept of the MPEG-I standard may or may not be used in this application. In medical applications, when an error occurs in image transmission, usually the whole image should be retransmitted. By removing the slice layer headers from the coded bit stream, the compression ratio improves by $0.5 \%$ in $\mathrm{CT}$ images with $\mathrm{CR}$ of approximately $10: 1$.

The 'macroblock' and 'block' layers exist in our algorithm similar to the MPEG-I standard. All the coding methods are kept the same; however new VLC codebooks are generated and used. The wellknown D-type pictures [1] which are low-resolution images that enable fast decompression for fast forward and reverse display purposes are not suitable for our purposes. The aim of this work is to show the use of predictive and motion-compensated compression in the medical applications only. The use of bidirectionally predicted B-type pictures may increase the compression gain; however it does not contribute to the results that we obtain for this work. Furthermore, there are works $[11,1]$ which describe some methods discarding the B-type frames without much compression loss. As a result, $B$ and D-type pictures are not used in our implementation.

In the block coding, we also modified the coding of rarely occurring (run, level) pairs to improve coding efficiency. Since the dynamic range of the medical pictures are 10 or 12 bits, the statistics of rarely occurring (run, level) pairs changes from those of 8-bit video images.

\section{Simulation examples and conclusion}

We used both objective and subjective performance measures for the evaluation of our 
compression method. We made comparisons with a single frame image compression method (using I-type frames only) which is basically a modified implementation of the JPEG algorithm [7].

The given test sequence contains $17512 \times 512$ sized abdominal CT images with resolution 10 bits per pixel. We used the PSNR,

PSNR $=-20 \log _{10}\left(\frac{\sigma}{1023}\right)$

where

$\sigma=\left[\frac{1}{512 \times 512} \sum_{i=0}^{511} \sum_{j=0}^{511}\left(x_{\text {orig }}(i, j)-x_{\text {comp }}(i, j)\right)^{2}\right]_{(2)}^{1 / 2}$

to evaluate the quality of the compressed pictures.

In this paper, results for two compression efficiency levels (compression ratios (CR) of approximately 10:1 and 20:1) are presented in Tables 1 and 2. In the last rows of Tables 1 and 2 , we gave the intra-frame only coding results. By comparing these rows with the rest of the tables, we observed that better compression ratios can be achieved by using more P-type pictures for a given PSNR value for a GOP with more than one P-type picture. We also note that the average PSNR tends to decrease as the number of consecutive P-type pictures increase in a GOP. This imposes a trade-off between the average $C R$ and the PSNR in choosing the GOP structure to be used. Based on our results the GOP structures IPPIPP... and IPPPIPPP... are the ones which balance these two features in a good way. Fixing the CR or PSNR values to an exact prescribed value is quite difficult for this scheme because the quantizer scale index (QSI) parameter modulating the quantizer step size assumes only integer values as in MPEG-I standard. However, in case of both Tables 1 and 2, the results for GOP structures IPP and IPPP show that for a given PSNR level using P-type pictures in the GOP structure enables a better compression ratio as compared to using only I-type coding. The gain in the compressed file is approximately $20 \mathrm{~K}$ bytes per compressed CT image sequence consisting of 20 images.

The image shown in Fig. 1 is an original image from the test sequence. Fig. 2 shows its I-type coded version $(C R=10.55: 1)$. In Fig. 3, another original
Table 1

Coding results for $\sim 10: 1 \mathrm{CR}$ level in our algorithm. Compression ratios and PSNR values are determined by using the ensamble of pictures in the GOP. The last row is the result of intra-frame only coding

\begin{tabular}{llc}
\hline GOP structure & Average PSNR & Average CR \\
\hline IP & 59.17 & 9.89 \\
IPP & 59.03 & 10.21 \\
IPPP & 58.93 & 10.27 \\
IPPPP & 58.80 & 10.23 \\
IPPPPP & 58.56 & 10.12 \\
IPPPPPP & 58.44 & 10.00 \\
II ... I & 58.90 & 9.96 \\
\hline
\end{tabular}

Table 2

Coding results for $\sim 20: 1 \mathrm{CR}$ level in our algorithm. Compression ratios and PSNR values are determined by using the ensamble of pictures in the GOP. The last row is the result of intra-frame only coding

\begin{tabular}{lll}
\hline GOP structure & Average PSNR & Average CR \\
\hline IP & 55.32 & 21.00 \\
IPP & 55.00 & 24.36 \\
IPPP & 54.85 & 23.20 \\
IPPPP & 54.54 & 22.56 \\
IPPPPP & 54.01 & 22.15 \\
IPPPPPP & 53.81 & 21.86 \\
II ... I & 54.59 & 21.40 \\
\hline
\end{tabular}

image from the same sequence is shown. The P-type coded version of this image at the $\mathrm{CR}=10.35: 1$ is shown in Fig. 4. The difference image produced by subtracting the original image from its P-type coded version ( $C R=22.56: 1)$ is shown in Fig. 5. In all the P-type coded pictures shown here, the GOP is in the form IPPPP and the picture is the last P-type picture.

As a subjective measure we resorted to the opinions of a group of medical doctors. This group contained one radiologist, one internal medicine expert and a practitioner. They evaluated the original and the compressed images in terms of (i) the overall picture quality, and (ii) the picture quality based on diagnostic features. The group stated that the compressed pictures with approximately 10:1 compression ratios cannot be distinguished from 


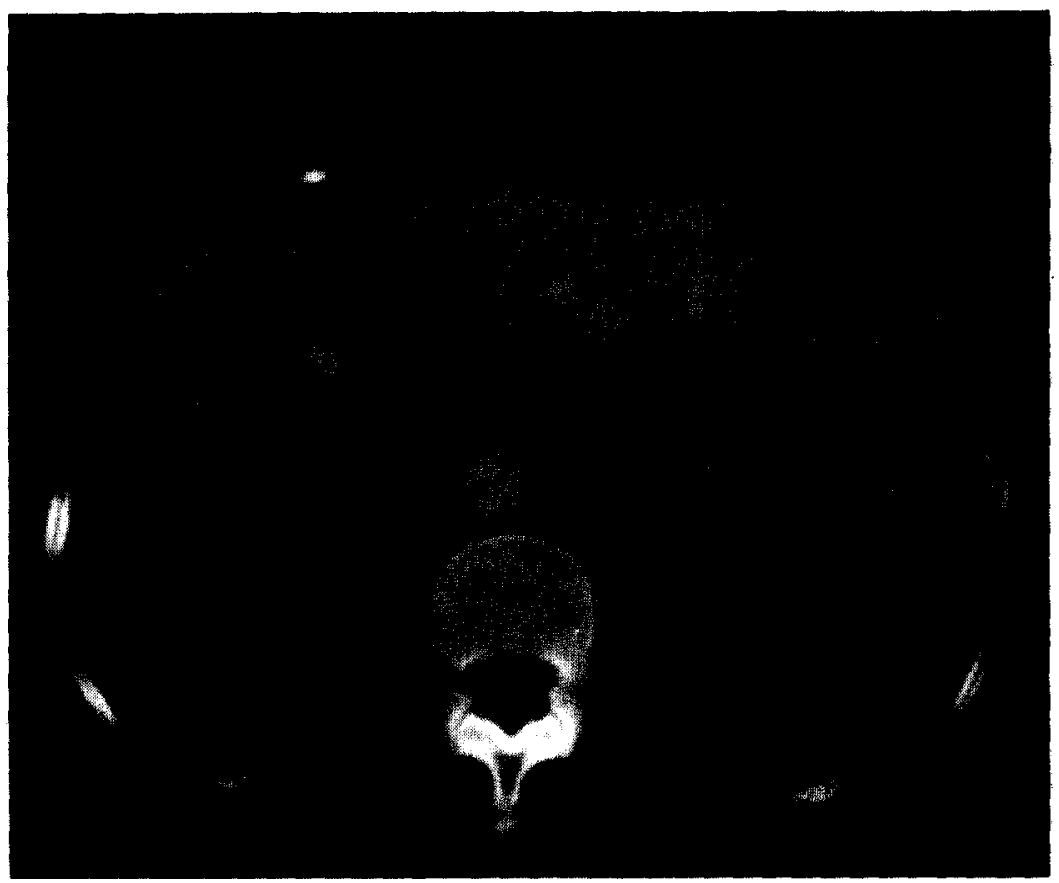

Fig. 1. An original image from the test sequence.

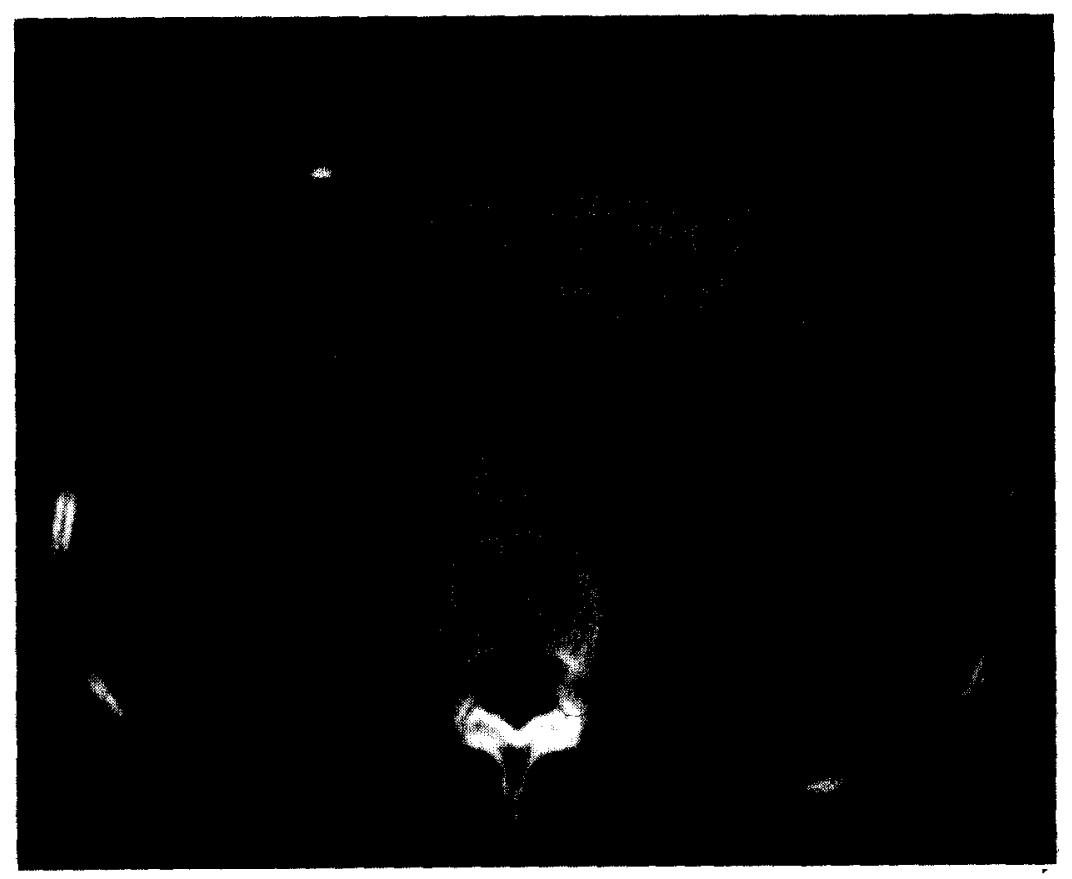

Fig. 2. The image in Fig. 1 after I-type coding at $C R=10.55: 1$ (0.95 bits/pel). 


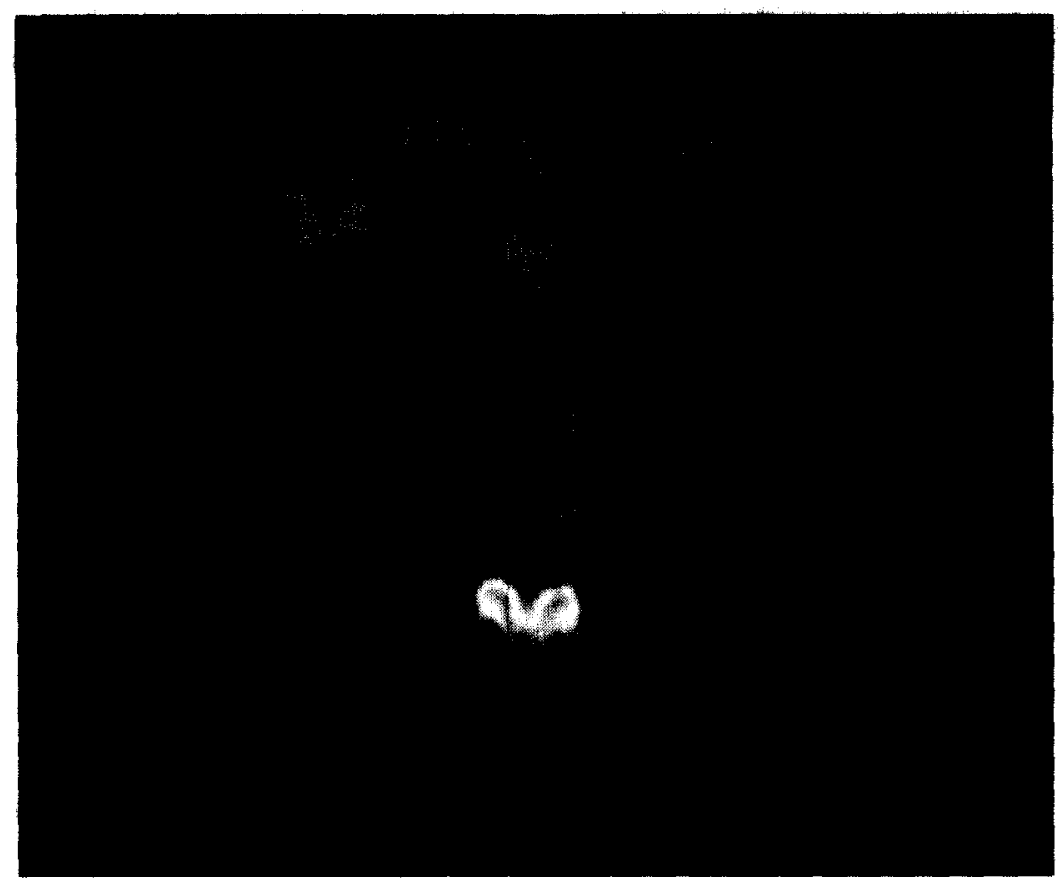

Fig. 3. Another original image from the test sequence.

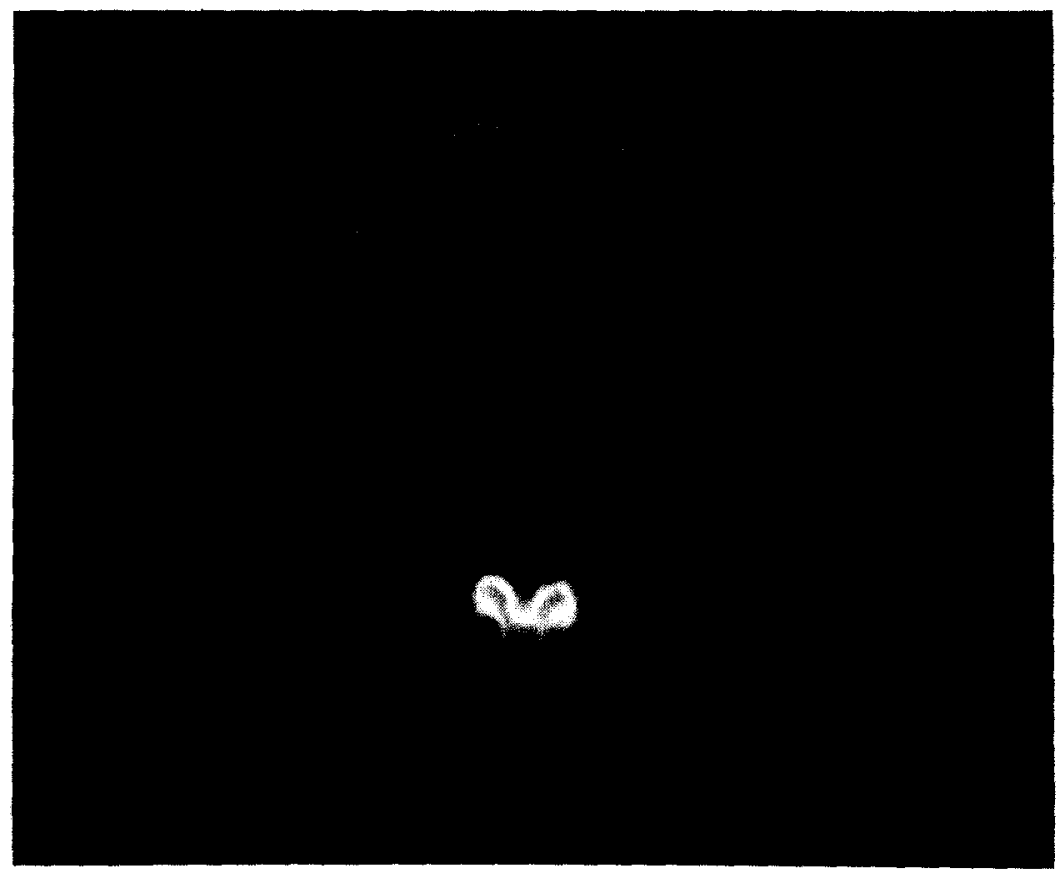

Fig. 4. The image in Fig. 3 after P-type coding at $C R=10.35: 1(0.97$ bits/pel). 


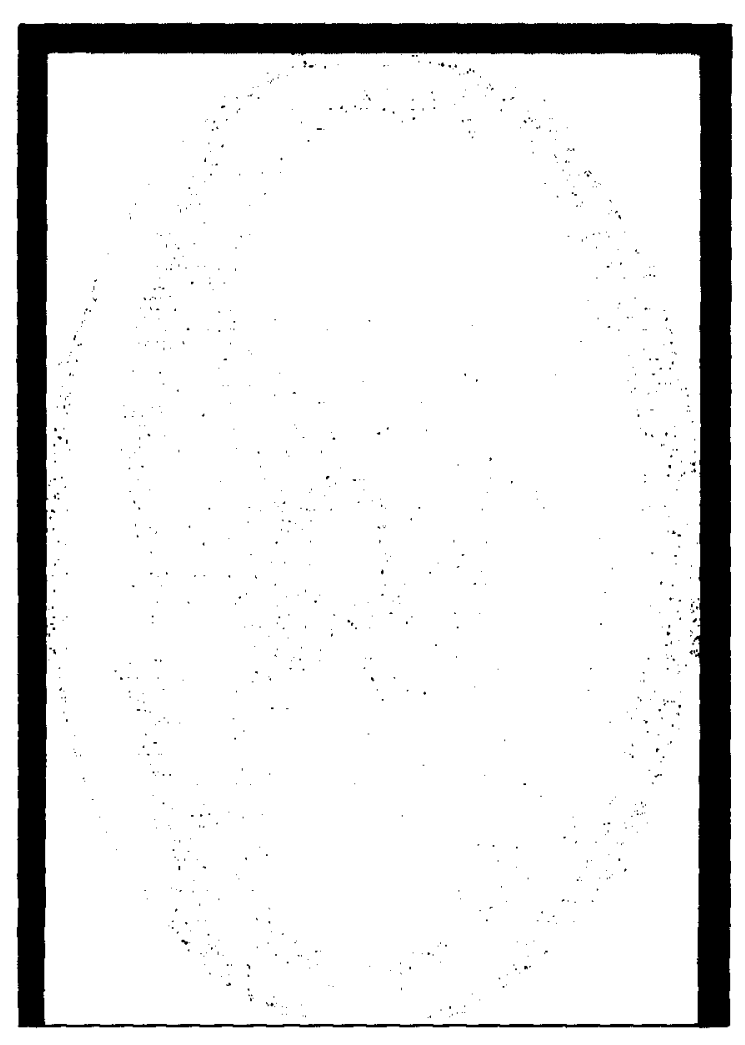

Fig. 5. The difference image $(12 \times$ [original - reconstructed]) for P-type coding at $\mathrm{CR}=22.56(0.44 \mathrm{bits} / \mathrm{pel})$. The darker the points $\mathrm{gct}$, the higher the difference is.

their originals. This is valid for both I- and P-type pictures in all GOP-types tested. However, there are visible degradations in approximately $20: 1$ compressed pictures which may still be used in a PACS for archiving purposes, if a radiologist's report accompanies the image sequence. Similar results can be obtained for other medical image sequences, too.

Three-dimensional coding of medical image sequences was first considered by Huang et al. [3]. In [3], a 3-D DCT-based coding method was developed. However, this method does not use 'motion-compensation' techniques which are widely utilized in video-sequence coding including MPEG-I [7]. In this paper, we experimentally observed that the use of predictive coding and motion-compensated coding in a medical image sequence compression increases the coding efficiency without compromising objective and subjective picture quality.

\section{References}

[1] G. Bjøntegaard, Proposal 06, VADIS/COST Forward Prediction Coding, MPEG91/270, November 1991.

[2] A.E. Cetin, "Subband coding of DSA images", in: H.K. Huang, O. Ratib, A.R. Bakker and G. Witte, eds., Picture Archiving and Communication Systems (PALS) in Medicine, Springer, Berlin, 1991, pp. 361-363.

[3] K.K. Chan, C.C. Lau, S.L, Lou, A. Hayrapetian, B.K.T. Ho and H.K. Huang, "Three-dimensional transform compression of images from dynamic studies", The UCLA PACS modules and related projects - A progress report, Los Angeles, CA, 1990, pp. 60-64.

[4] P.S. Cho, K.K. Chan and K.T. Ho, "Data storage and compression", in: H.K. Huang, O. Ratib, A.R. Bakker and G. Witte, eds., Picture Archiving and Communication Systems (PALS) in Medicine, Springer, Berlin, 1991, pp. 71-82.

[5] H.K. Huang, O. Ratib, A.R. Bakker and G. Witte, eds., Picture Archiving and Communication Systems (PACS) in Medicine, Springer, Berlin, 1991.

[6] N.S. Jayant and P. Noll, Digital Coding of Waveforms, Prentice-Hall, Englewood Cliffs, NJ, 1984, pp. 320, 321.

[7] D. Le Gall, "Digital multimedia systems: Digital image and video standards", Commun. AC.M, Vol. 34, 1991, pp. 47-58.

[8] MPEG-I standard ISO/IEC IS11172.

[9] M. Rabbani and P.W. Jones, Digital image Compression Techniques, SPIE Press, USA, 1991.

[10] T.V. Ramabadran and K. Chen, "The use of contextual information in the reversible compression of medical images", IEEE Trans. Medical Imaging, Vol. 11, No. 2, June 1992, pp. 185-195.

[11] H. Sandgrind, NTR proposal to MPEG-2/Video tests, Kurihama, Japan, 1992.

[12] W.S. Weinberg, M. Loloyan, R.K. Taira, K.K. Chan and H.K. Huang, "Automatic acquisition of CT, MR, and US images for PACS", in: H.K. Huang, O. Ratib, A.R. Bakker and G. Witle, eds., Picture Archiving and Communication Systems (PALS) in Medicine, Springer, Berlin, 1991, pp. 43-50.

[13] P. Wilhelm, D.R. Haynor, Y. Kim and E.A. Riskin, "Lossy image compression for digital medical imaging systems", SPIE Opt. Engrg., Vol, 30, October 1991, pp. 1479-1485.

[14] Y.Q. Zhang, M.H. Loew and R.L. Pickholtz, "A combined - transform coding (CTC) scheme for medical images", IEEE Trans. Medical Imaging, Vol. 11, No. 2, June 1992, pp. 196-202. 